\title{
SOME EXISTENCE THEOREMS IN THE CALCULUS OF VARIATIONS
}

\section{THE DRESDEN CORNER CONDITION*}

BY

\section{E. J. McSHANE}

The present note is the first of a series, and is the only one which does violence to the general title by failing to exhibit an existence theorem. Instead, we here perform a few rather easy computations for later use, and by them obtain a simple proof of a corner condition for isoperimetric problems. This corner condition was apparently first established by Dresden as a consequence of the Weierstrass condition, so only the method of proof here can qualify as new. However, it will serve as a suggestive guide to further theorems in later papers.

1. Notation and continuity assumptions. In order that some of our preliminary calculations and lemmas shall be valid for both parametric and non-parametric problems, we shall recast non-parametric problems in parametric form. Given an integrand

$$
f\left(x, y, y^{\prime}\right) \equiv f\left(x, y^{1}, \cdots, y^{q}, y^{1}, \cdots, y^{q^{\prime}}\right),
$$

we define

$$
\begin{aligned}
F\left(z, z^{\prime}\right) & =F\left(z^{0}, z^{1}, \cdots, z^{\dot{q}}, z^{0^{\prime}}, z^{1^{\prime}}, \cdots, z^{q^{\prime}}\right) \\
& =z^{0^{\prime}} f\left(z^{0}, z^{1}, \cdots, z^{q} ; \frac{z^{1^{\prime}}}{z^{0^{\prime}}}, \cdots, \frac{z^{q^{\prime}}}{z^{0^{\prime}}}\right), \quad z^{0^{\prime}}>0, \\
F(z, 0) & =0 .
\end{aligned}
$$

We use a modification of the tensor summation convention. The repetition of a Greek-letter affix in a term requires the summation of the values of that term over all values of the affix. Thus

$$
F_{z^{\alpha}}\left(z_{n}, z_{n}^{\prime}\right) z^{\alpha^{\prime}}=F_{z 0}\left(z_{n}, z_{n}^{\prime}\right) z^{0 \prime}+\cdots+F_{z^{q}}\left(z_{n}, z_{n}^{\prime}\right) z^{q^{\prime}}
$$

is summed over $\alpha=0,1, \cdots, q$, but not summed over the values of $n$. Also, if $\nu$ is a vector, its length will be denoted by $|\nu|$. Thus if $z=\left(z^{0}, \cdots, z^{q}\right)$, then $|z|=\left[z^{\alpha} z^{\alpha}\right]^{1 / 2}$.

* Presented to the Society, December 28, 1937; received by the editors October 29, 1937 and, in revised form, February 16, 1938. 
Throughout the papers of this series all integrands $f\left(x, y, y^{\prime}\right)$ will be assumed (unless specific statement is made to the contrary) to be defined and continuous together with their partial derivatives of first and second order for all $(x, y)$ in a closed set $S$, and all $y^{\prime}$. Furthermore, we assume without further mention the following:

(1.2) For every bounded subset $S_{0}$ of $S$ there is a constant $N$ such that

$$
f\left(x, y, y^{\prime}\right)+N\left[1+y^{\alpha^{\prime}} y^{\alpha^{\prime}}\right]^{1 / 2} \geqq 0
$$

for all $(x, y)$ in $S_{0}$ and all $y^{\prime}$.

In the parametric notation, $F\left(z, z^{\prime}\right)$ being defined by (1.1), this takes the form:

$\left(1.2^{\prime}\right)$ For every bounded subset $S_{0}$ of $S$ there is a constant $N$ such that $F\left(z, z^{\prime}\right)+N\left|z^{\prime}\right| \geqq 0$ for all $z$ in $S_{0}$ and all $z^{\prime}$ with $z^{0 \prime}>0$.

Parametric integrands $F\left(z, z^{\prime}\right)$ (not those arising by (1.1) from nonparametric integrands) will be assumed to be defined and continuous for all $z$ in a closed set $S$ and all $z^{\prime}$, to be positively homogeneous of degree 1 in $z^{\prime}$, and to have continuous partial derivatives of the first and second orders for all $z$ in $S$ and for all $z^{\prime} \neq(0, \cdots, 0)$.

In order to avoid frequent printing of a complicated symbol we make the definitions

$$
\begin{aligned}
f_{i}\left(x, y, y^{\prime}\right) & \equiv f_{y^{i}}\left(x, y, y^{\prime}\right), & i=1, \cdots, q, \\
F_{i}\left(z, z^{\prime}\right) & \equiv F_{z^{i}},\left(z, z^{\prime}\right), & i=0,1, \cdots, q .
\end{aligned}
$$

From the assumptions made on integrands $f\left(x, y, y^{\prime}\right)$ it follows at once that the function $F\left(z, z^{\prime}\right)$ defined by (1.1) is continuous with its partial derivatives of first and second orders for all $z \equiv(x, y)$ in $S$ and all $z^{\prime}$ with $z^{0 \prime}>0$, and is positively homogeneous of degree 1 in $z^{\prime}$. Whenever we are discussing the integral of such an integrand along a curve $C: z=z(t)$ it will be tacitly assumed that $z^{\prime \prime}(t)>0$ for almost all $t$, while for all auxiliary curves used in demonstrations we must prove $z^{0 \prime}(t)>0$ for almost all $t$.

If the representation $z=z(t),\left(t_{1} \leqq t \leqq t_{2}\right)$, of the curve $C$ satisfies this condition, the function* $F(z(t), \dot{z}(t))$ is measurable. Since by $\left(1.2^{\prime}\right)$ it exceeds a summable function $-N|\dot{z}(t)|$, it has an integral, finite or infinite. Provided that the functions $z^{i}(t)$ are absolutely continuous, we denote this integral by

$$
\mathcal{F}(C)=\int_{C} F(z, \dot{z}) d t=\int_{t_{1}}^{t_{2}} F(z(t), \dot{z}(t)) d t .
$$

* In analogy with Carathéodory's notation, we define $\dot{z}(t)$ to be the vector $\left(z^{0^{\prime}}(t), \cdots, z^{q^{\prime}}(t)\right)$ if the vector is defined and finite; otherwise $\dot{z}(t)$ is defined to be 0 (i.e., $(0, \cdots, 0)$ ). 
Any two absolutely continuous parametrizations of $C$ (subject to the requirement that $z^{0 \prime}(t)>0$ for almost all $t$ ) give the same value to the integral.* In particular, if $z^{0}(t) \equiv t$, so that $C$ has the form $y=y(x),(a \leqq x \leqq b)$, and if furthermore the $y^{i}(x)$ are absolutely continuous, we write

$$
\mathcal{F}[y]=\mathcal{F}(C)=\int_{a}^{b} f(x, y(x), \dot{y}(x)) d x .
$$

If $F\left(z, z^{\prime}\right)$ is a parametric integrand, defined for all $z$ in a set $S$ and all $z^{\prime}$, these questions of integrability do not arise. We write in this case also

$$
\mathcal{F}(C)=\int_{C} F(z, \dot{z}) d t=\int_{t_{1}}^{t_{2}} F(z(t), \dot{z}(t)) d t,
$$

provided that the functions $z^{i}(t)$ are absolutely continuous. The invariance under change of parameter is well known in this case. (It also follows from the theorem cited in the preceding footnote.)

2. Differentiation formulas. Let $z=z(\sigma),\left(\sigma_{1} \leqq \sigma \leqq \sigma_{2}\right)$, be a Lipschitzian representation of a curve $C$. We shall suppose that $z=\phi(\tau)$ and $z=\psi(\tau)$ are two curves passing through the ends of $C$, so that

$$
\phi\left(\tau_{0}\right)=z\left(\sigma_{1}\right), \quad \psi\left(\tau_{0}\right)=z\left(\sigma_{2}\right),
$$

and we shall also suppose that $\phi$ and $\psi$ are absolutely continuous on an interval $(a, b)$ containing $\tau_{0}$ and have finite derivatives for $\tau=\tau_{0}$.

From $C$ we form the curve $C(\tau)$ defined by the equation $\dagger$

$$
\begin{aligned}
C(\tau): z^{i}= & z^{i}(\sigma, \tau)=z^{i}(\sigma)+\frac{\sigma_{2}-\sigma}{\sigma_{2}-\sigma_{1}}\left(\phi^{i}(\tau)-\phi^{i}\left(\tau_{0}\right)\right) \\
& +\frac{\sigma-\sigma_{1}}{\sigma_{2}-\sigma_{1}}\left(\psi^{i}(\tau)-\psi^{i}\left(\tau_{0}\right)\right), \sigma_{1} \leqq \sigma \leqq \sigma_{2}, i=0,1, \cdots, q .
\end{aligned}
$$

This (by (2.1)) joins $\phi(\tau)$ to $\psi(\tau)$, and $z\left(\sigma, \tau_{0}\right) \equiv z(\sigma)$. We wish to calculate the derivative of $\mathcal{F}(C(\tau))$, it being assumed that $C(\tau)$ lies in $S$ for $a \leqq \tau \leqq b$.

Since

$$
\mathcal{F}(C(\tau))=\int_{\sigma_{1}}^{\sigma_{2}} F(z(\sigma, \tau), \dot{z}(\sigma, \tau)) d \sigma
$$

by differentiating under the integral sign we obtain

* E. J. McShane, Semi-continuity of integrals in the calculus of variations, Duke Mathematical Journal, vol. 2 (1936), pp. 597-616; in particular, Theorem 2.1 (the first five lines of proof being deleted).

$\dagger$ The curve $C(\tau)$ depends not merely on $C$ and $\tau$, but also on the particular parametrization $z=z(\sigma)$ which we chose for $C$. 


$$
\begin{aligned}
\left.\mathcal{F}^{\prime}(C(\tau))\right|_{\tau=\tau_{0}}= & \left.\int_{\sigma_{1}}^{\sigma_{2}}\left(F_{z^{\alpha}} \frac{\partial z^{\alpha}}{\partial \tau}+F_{\alpha} \frac{\partial \dot{z}^{\alpha}}{\partial \tau}\right) d \sigma\right|_{\tau=\tau_{0}} \\
= & \int_{\sigma_{1}}^{\sigma_{2}}\left\{F_{z^{\alpha}}(z(\sigma), \dot{z}(\sigma))\left[\frac{\sigma_{2}-\sigma}{\sigma_{2}-\sigma_{1}} \phi^{\alpha^{\prime}}\left(\tau_{0}\right)+\frac{\sigma-\sigma_{1}}{\sigma_{2}-\sigma_{1}} \psi^{\alpha^{\prime}}\left(\tau_{0}\right)\right]\right. \\
& \left.+F_{\alpha}(z(\sigma), \dot{z}(\sigma))\left[\frac{\psi^{\alpha^{\prime}}\left(\tau_{0}\right)-\phi^{\alpha^{\prime}}\left(\tau_{0}\right)}{\sigma_{2}-\sigma_{1}}\right]\right\} d \sigma .
\end{aligned}
$$

If $F\left(z, z^{\prime}\right)$ arises from a non-parametric integrand by (1.1), equation (2.4) is still valid if we add the hypothesis that $\dot{z}^{0}(\sigma) \geqq m>0$ for almost all $\sigma$.

Several special cases of this formula will be of use to us. First, suppose $\phi(\tau)-\phi\left(\tau_{0}\right)=\psi(\tau)-\psi\left(\tau_{0}\right)$, so that (2.2) represents merely a translation of the curve $C$ by the (vector) amount $\phi(\tau)-\phi\left(\tau_{0}\right)$. Then

$$
\mathcal{F}^{\prime}\left(C\left(\tau_{0}\right)\right)=\phi^{\alpha^{\prime}}\left(\tau_{0}\right) \int_{\sigma_{1}}^{\sigma_{2}} F_{z^{\alpha}}(z, \dot{z}) d \sigma .
$$

(This is independent of the parametric representation of $C$.)

Suppose next that $C$ is a line segment, and that $C(\tau)$ is the line segment whose ends are $z\left(\sigma_{1}\right)+\tau \pi_{1}$ and $z\left(\sigma_{2}\right)+\tau \pi_{2}$, where $\pi_{1}$ and $\pi_{2}$ are given vectors. If the functions $z=z(\sigma)$ representing $C$ are linear, then (2.2) represents the line segment $C(\tau)$ if we take

$$
\phi(\tau)=z\left(\sigma_{1}\right)+\tau \pi_{1} \text { and } \psi(\tau)=z\left(\sigma_{2}\right)+\tau \pi_{2},
$$

so that (2.4) becomes

$$
\begin{aligned}
\left.\mathcal{F}^{\prime}(C(\tau))\right|_{\tau=r_{0}}= & \int_{\sigma_{1}}^{\sigma_{2}}\left\{F_{z^{\alpha}}(z, \dot{z})\left[\frac{\sigma_{2}-\sigma}{\sigma_{2}-\sigma_{1}} \pi_{1}^{\alpha}+\frac{\sigma-\sigma_{1}}{\sigma_{2}-\sigma_{1}} \pi_{2}^{\alpha}\right]\right. \\
& \left.+F_{\alpha}(z, \dot{z})\left[\frac{\pi_{2}^{\alpha}-\pi_{1}^{\alpha}}{\sigma_{2}-\sigma_{1}}\right]\right\} d \sigma .
\end{aligned}
$$

Applying the mean value theorem to the last term on the right, we obtain

$$
\begin{aligned}
\left.\mathcal{F}^{\prime}(C(\tau))\right|_{\tau=\tau_{0}}= & \int_{\sigma_{1}}^{\sigma_{2}} F_{z^{\alpha}}(z(\sigma), \dot{z}(\sigma))\left\{\pi_{1}^{\alpha} \frac{\sigma_{2}-\sigma}{\sigma_{2}-\sigma_{1}}+\pi_{2}^{\alpha} \frac{\sigma-\sigma_{1}}{\sigma_{2}-\sigma_{1}}\right\} d \sigma \\
& +F_{\alpha}(z(\bar{\sigma}), \dot{z}(\bar{\sigma}))\left[\pi_{2}^{\alpha}-\pi_{1}^{\alpha}\right],
\end{aligned}
$$

where $\sigma_{1}<\bar{\sigma}<\sigma_{2}$.

3. Interchange of arcs. Let $C_{1}$ and $C_{2}$ be rectifiable curves

$$
C_{1}: \quad z=z_{1}(\sigma), \sigma_{1} \leqq \sigma \leqq \sigma_{2} ; \quad C_{2}: \quad z=z_{2}(\tau), \tau_{1} \leqq \tau \leqq \tau_{2}
$$

such that the beginning of $C_{2}$ coincides with the end of $C_{1}$ :

$$
z_{1}\left(\sigma_{2}\right)=z_{2}\left(\tau_{1}\right) .
$$


The functions $z_{1}(\sigma)$ and $z_{2}(\tau)$ will be supposed to satisfy a Lipschitz condition, and if $F\left(z, z^{\prime}\right)$ is defined by (1.1) it will also be supposed that except on a set of measure zero $\dot{z}_{1}{ }^{0}(t)$ and $\dot{z}_{2}{ }^{0}(t)$ are bounded from zero. We define $C_{12}$ to be the curve obtained by traversing first $C_{1}$ and then $C_{2}$. Furthermore, we define $C_{21}$ to be the curve obtained by starting at $z_{1}\left(\sigma_{1}\right)$, traversing a curve $C_{2}^{*}$ which is a translation of $C_{2}$, and then traversing a curve $C_{1}^{*}$ which is a translation of $C_{1}$. It is clear that the end of $C_{21}$ is the same as the end of $C_{12}$, namely $z_{2}\left(\tau_{2}\right)$. We now assume that the point

$$
z_{1}(\sigma)+z_{2}(\tau)-z_{2}\left(t_{1}\right)
$$

lies in $S$ whenever $\sigma_{1} \leqq \sigma \leqq \sigma_{2}$ and $\tau_{1} \leqq \tau \leqq \tau_{2}$, and we proceed to compute $\mathcal{f}\left(C_{21}\right)-\mathcal{F}\left(C_{12}\right)$.

By the definitions,

$$
\mathcal{f}\left(C_{21}\right)-\mathcal{F}\left(C_{12}\right)=\mathcal{F}\left(C_{1}^{*}\right)-\mathcal{F}\left(C_{1}\right)+\mathcal{F}\left(C_{2}^{*}\right)-\mathcal{F}\left(C_{2}\right) .
$$

We compute $\mathcal{F}\left(C_{1}^{*}\right)-\mathcal{F}\left(C_{1}\right)$ by $(2.5)$, where we take $\phi(\tau)=z_{2}(\tau)-z_{1}\left(\sigma_{2}\right)+z_{1}\left(\sigma_{1}\right)$, $\psi(\tau)=z_{2}(\tau)$. If we momentarily let $C(\tau)$ be the curve (which by hypothesis lies in $S), z=z_{1}(\sigma)+z_{2}(\tau)-z_{2}\left(\tau_{1}\right),\left(\sigma_{1} \leqq \sigma \leqq \sigma_{2}\right)$, we obtain by $(2.5)$

$$
\mathcal{F}^{\prime}(C(\tau))=\dot{z}_{2}^{\alpha}(\tau) \int_{\sigma_{1}}^{\sigma_{2}} F_{z^{\alpha}}\left(z_{1}(\sigma)+z_{2}(\tau)-z_{2}\left(\tau_{1}\right), \dot{z}_{1}(\sigma)\right) d \sigma
$$

for all $\tau$ such that $z_{2}^{\prime}(\tau)$ exists, that is, for almost all $\tau$. Hence, integrating from $\tau=\tau_{1}$ to $\tau=\tau_{2}$, we obtain

$$
\begin{aligned}
\mathcal{f}\left(C_{1}^{*}\right)-\mathcal{F}\left(C_{1}\right) & =\mathcal{F}\left(C\left(\tau_{2}\right)\right)-\mathcal{f}\left(C\left(\tau_{1}\right)\right) \\
& =\int_{\tau_{1}}^{\tau_{2}} \int_{\sigma_{1}}^{\sigma_{2}} \dot{z}_{2}^{\alpha}(\tau) F_{z^{\alpha}}\left(z_{1}(\sigma)+z_{2}(\tau)-z_{2}\left(\tau_{1}\right), \dot{z}_{1}(\sigma)\right) d \sigma d \tau .
\end{aligned}
$$

Next we let $C^{\prime}(\sigma)$ be the curve $z=z_{2}(\tau)+z_{1}(\sigma)-z_{1}\left(\sigma_{2}\right),\left(\tau_{1} \leqq \tau \leqq \tau_{2}\right)$. (Recalling (3.2), we see that this lies by hypothesis in $S$ for $\sigma_{1} \leqq \sigma \leqq \sigma_{2}$.) Then $C^{\prime}\left(\sigma_{2}\right)$ is $C_{2}$; and $C^{\prime}\left(\sigma_{1}\right)$ is $C_{2}^{*}$, for it is a translation of $C_{2}$ and it starts at $z_{2}\left(\tau_{1}\right)+z_{1}\left(\sigma_{1}\right)-z_{1}\left(\sigma_{2}\right)$, which is $z_{1}\left(\sigma_{1}\right)$ by (3.2). Again using (2.5), but with $\sigma$ serving in the role of $\tau$, we find

$$
\mathcal{f}^{\prime}\left(C^{\prime}(\sigma)\right)=\dot{z}_{1}^{\alpha}(\sigma) \int_{\tau_{1}}^{\tau_{2}} F_{z^{\alpha}}\left(z_{2}(\tau)+z_{1}(\sigma)-z_{1}\left(\sigma_{2}\right), \dot{z}_{2}(\tau)\right) d \tau .
$$

Integrating from $\sigma_{1}$ to $\sigma_{2}$, we have

$$
\begin{aligned}
\mathcal{F}\left(C_{2}\right)-\mathcal{F}\left(C_{2}^{*}\right) & =\mathcal{F}\left(C^{\prime}\left(\sigma_{2}\right)\right)-\mathcal{F}\left(C^{\prime}\left(\sigma_{1}\right)\right) \\
& =\int_{\sigma_{1}}^{\sigma_{2}} \int_{\tau_{1}}^{\tau_{2}} \dot{z}_{1}^{\alpha}(\sigma) F_{z^{\alpha}}\left(z_{2}(\tau)+z_{1}(\sigma)-z_{1}\left(\sigma_{2}\right), \dot{z}_{2}(\tau)\right) d \tau d \sigma .
\end{aligned}
$$


With Carathéodory, we now define

$$
\Omega(z, p, q)=p^{\alpha} F_{z^{\alpha}}(z, q)-q^{\alpha} F_{z^{\alpha}}(z, p) .
$$

Then from (3.4), (3.6), and (3.8) we obtain (recalling (3.2))

(3.10) $\mathcal{f}\left(C_{21}\right)-\mathcal{F}\left(C_{12}\right)=-\int_{\tau_{1}}^{\tau_{2}} \int_{\sigma_{1}}^{\sigma_{2}} \Omega\left(z_{2}(\tau)+z_{1}(\sigma)-z_{1}\left(\sigma_{2}\right), \dot{z}_{1}(\sigma), \dot{z}_{2}(\tau)\right) d \sigma d \tau$.

We digress for a moment to make some remarks on the $\Omega$-function. It is clear that it is continuous for all $z, p$, and $q$, if $F$ is the usual parametric integrand. If $F$ arises by (1.1), then $\Omega$ is continuous if $p^{0}>0$ and $q^{0}>0$. From the definition, $\Omega(z, p, q)$ is positively homogeneous of degree 1 in $p$ and in $q$ :

$$
\Omega(z, k p, \kappa q)=k \kappa \Omega(z, p, q) \text { if } k>0 \text { and } \kappa>0 .
$$

Also

$$
\Omega(z, p, q)=-\Omega(z, q, p) .
$$

Suppose that $F\left(z, z^{\prime}\right)$ arises by (1.1) from $f\left(x, y, y^{\prime}\right)$, where $\left(z^{0}, z^{1}, \cdots, z^{q}\right)$ $=\left(x, y^{1}, \cdots, y^{q}\right)$, and that the curves $C_{1}, C_{2}$ are represented in the form

$$
C_{1}: \quad y=y_{1}(x), a \leqq x \leqq b ; \quad C_{2}: \quad y=y_{2}(x), b \leqq x \leqq c,
$$

where $y_{1}(b)=y_{2}(b)$. We can if we wish revert to the integrand $f$ by simply using $x$ as parameter (it being still assumed that $y_{1}(x)$ and $y_{2}(x)$ are Lipschitzian). Then on $C_{1}$ we have $\sigma=x$; so $z_{1}{ }^{0}(\sigma)=\sigma=x$, and $\dot{z}_{1}{ }^{0}(\sigma) \equiv 1$. Likewise $\dot{z}_{2}{ }^{0}(\tau) \equiv 1$, so that by $(3.10)$

$$
\begin{aligned}
& \mathcal{f}\left(C_{21}\right)-\mathcal{F}\left(C_{12}\right) \\
& =-\int_{b}^{c}\left\{\int_{a}^{b} \omega\left(\sigma+\tau-a, y_{1}(\sigma)+y_{2}(\tau)-y_{1}(b), \dot{y}_{1}(\sigma), \dot{y}_{2}(\tau)\right) d \sigma\right\} d \tau,
\end{aligned}
$$

where

$$
\omega\left(x, y, y^{\prime}, Y^{\prime}\right)=f_{x}\left(x, y, Y^{\prime}\right)-f_{x}\left(x, y, y^{\prime}\right)
$$

$$
+y^{\alpha^{\prime}} f_{y^{\alpha}}\left(x, y, Y^{\prime}\right)-Y^{\alpha^{\prime}} f_{y^{\alpha}}\left(x, y, y^{\prime}\right)
$$

that is,

$$
\omega\left(x, y, y^{\prime}, Y^{\prime}\right)=\Omega\left(x, y ; 1, y^{\prime} ; 1, Y^{\prime}\right) .
$$

As a special case (useful later) we may assume that $C_{1}$ and $C_{2}$ are line segments. If $l_{i}=L\left(C_{i}\right)$ is the length of $C_{i},(i=1,2)$, we can represent $C_{1}$ and $C_{2}$ by means of linear functions of arc lengths:

$$
C_{i}: \quad z=z_{i}(s),
$$$$
0 \leqq s \leqq l_{i}, i=1,2 \text {. }
$$

Then the derivative $\dot{z}_{i}(s)$ is constantly equal to a unit vector $p_{i},(i=1,2)$, 
and the curves $C_{12}$ and $C_{21}$ bound a parallelogram (which may degenerate into a line segment). Formula (3.10) becomes

$$
\mathcal{F}\left(C_{21}\right)-\mathcal{F}\left(C_{12}\right)=-\int_{0}^{l_{1}}\left\{\int_{0}^{l_{2}} \Omega\left(z_{1}(\sigma)+z_{2}(\tau)-z_{2}(0), p_{1}, p_{2}\right) d \tau\right\} d \sigma
$$

By the theorem of mean value,

$$
\mathcal{F}\left(C_{21}\right)-\mathcal{F}\left(C_{12}\right)=-l_{1} l_{2} \Omega\left(z_{1}(\bar{\sigma})+z_{2}(\bar{\tau})-z_{2}(0), p_{1}, p_{2}\right),
$$

where $0<\bar{\sigma}<l_{1}$ and $0<\bar{\tau}<l_{2}$. If we denote $z_{1}(\bar{\sigma})+z_{2}(\bar{\tau})-z_{2}(0)$ by $\xi$, then $\xi$ is a point of the parallelogram bounded by $C_{12}$ and $C_{21}$, and (3.18) becomes

$$
\mathcal{f}\left(C_{21}\right)-\mathcal{F}\left(C_{12}\right)=-l_{1} l_{2} \Omega\left(\xi, p_{1}, p_{2}\right) \text {. }
$$

4. Dresden's corner condition: the parametric form. In $\$ 4$ we establish Dresden's corner condition for isoperimetric problems in parametric form.

We shall proceed under the following hypotheses:

(4.1) The curve $C: z=z(t),\left(t_{1} \leqq t \leqq t_{2}\right)$, is interior to $S$ and gives a strong relative minimum to the integral

$$
\mathcal{F}(C)=\int_{C} F(z, \dot{z}) d t
$$

in the class of all $D^{\prime}$ curves which join $z\left(t_{1}\right)$ to $z\left(t_{2}\right)$ and give assigned values $\gamma_{j},(j=1, \cdots, m)$, to the integrals

$$
G^{j}(C) \equiv \int_{C} G^{j}(z, \dot{z}) d t, \quad j=1, \cdots, m .
$$

(4.2) The curve $C$ has a corner at $z\left(t_{0}\right)$.

(4.3) The curve $C$ is normal.* (Hence there is a unique set of multipliers $1, l_{1}, \cdots, l_{m}$ such that for the function

$$
H\left(z, z^{\prime}\right) \equiv F\left(z, z^{\prime}\right)+l_{\alpha} G^{\alpha}\left(z, z^{\prime}\right)
$$

the first variation of $\int_{C} H(z, z) d t$ vanishes along $C$.)

Then we have the theorem:

THEOREM 1. Under the hypotheses (4.1), (4.2), and (4.3) the inequality $\dagger$

$$
\Omega_{H}\left(z\left(t_{0}\right), z^{\prime}\left(t_{0}-0\right), z^{\prime}\left(t_{0}+0\right)\right) \leqq 0
$$

holds.

* We do not require normality on subarcs of $C$.

$\dagger$ Here, where several integrands are involved, there are several possible $\Omega$-functions. The subscript indicates the integrand used in defining $\Omega$ by (3.9). An analogous notation will be used for the $\varepsilon_{\text {-function. }}$ 
For compactness, we define

$$
p \equiv z^{\prime}\left(t_{0}-0\right), \quad r \equiv z^{\prime}\left(t_{0}+0\right) .
$$

Suppose that (4.4) fails to hold; then

$$
\Omega_{H}\left(z\left(t_{0}\right), p, r\right)>0 .
$$

By continuity, there is a positive number $\delta$, so small that $C$ has no corner other than $z\left(t_{0}\right)$ on the arc $z=z(t), t_{0}-\delta \leqq t \leqq t_{0}+\delta$, for which

$$
\Omega_{H}\left(z(\bar{t}), z^{\prime}(\bar{t}), r\right)>0 \text { if } t_{0}-\delta \leqq \bar{t}<t_{0} .
$$

Define $C_{r, 0}$ to be the curve obtained from $C$ by interchanging the arcs $z=z(t),\left(t_{0}-\delta \leqq t \leqq t_{0}\right)$, and $z=z(t),\left(t_{0} \leqq t \leqq t_{0}+\tau\right)$, where $0 \leqq \tau \leqq \delta$. The curve $C_{\tau, 0}$ is then defined by equations $z=\zeta_{\tau}(t),\left(t_{1} \leqq t \leqq t_{2}\right)$, where

$$
\begin{array}{ll}
\zeta_{\tau}(t)=z(t), \quad t_{1} \leqq t<t_{0}-\delta, & \\
\zeta_{\tau}(t)=z(t+\delta)-z\left(t_{0}\right)+z\left(t_{0}-\delta\right), & t_{0}-\delta \leqq t<t_{0}-\delta+\tau, \\
\zeta_{\tau}(t)=z(t-\tau)-z\left(t_{0}\right)+z\left(t_{0}+\tau\right), & t_{0}-\delta+\tau \leqq t<t_{0}+\tau, \\
\zeta_{\tau}(t)=z(t), \quad t_{0}+\tau \leqq t \leqq t_{2} . &
\end{array}
$$

(Thus $C_{0,0}$ coincides with $C$. For $\tau$ near 0 , it is clear that $C_{\tau, 0}$ is in $S$.)

Given any set of $m$ (vector) functions $\eta_{j}(t)=\left(\eta_{j}{ }^{0}(t), \cdots, \eta_{j}{ }^{q}(t)\right)$ of class $D^{\prime}$ on $\left[t_{1}, t_{2}\right]$ and vanishing at $t_{1}$ and $t_{2}$, we shall define

$$
\begin{aligned}
\Phi(\tau, b) & \equiv \Phi\left(\tau, b_{1}, \cdots, b_{m}\right) \equiv \mathcal{F}\left(C_{\tau, b_{1}, \cdots, b_{m}}\right), \\
\Gamma^{i}(\tau, b) & \equiv \Gamma^{j}\left(\tau, b_{1}, \cdots, b_{m}\right) \equiv \mathcal{G}^{i}\left(C_{r, b_{1}, \cdots, b_{m}}\right),
\end{aligned}
$$

where $C_{\tau, b}$ is given by the following:

(4.10) $C_{\tau, b} \equiv C_{\tau, b_{1}}, \cdots_{b_{m}}$ is defined by $z=z(t ; \tau, b) \equiv \zeta_{\tau}(t)+b_{\alpha} \eta_{\alpha}(t) ;\left(t_{1} \leqq t \leqq t_{2}\right)$.

For $\tau$ and $b$ near 0 , this lies in $S$. As we know,

$$
\left.\frac{\partial \Gamma^{j}}{\partial b_{\kappa}}\right|_{\tau=b=0}=\int_{t_{1}}^{t_{2}} \eta_{\kappa}^{\alpha}(t)\left\{-\frac{d}{d t} G_{\alpha}^{j}(z, \dot{z})+G_{z^{\alpha}}^{j}(z, \dot{z})\right\} d t .
$$

We have assumed that $C$ is normal. Hence there exist functions $\eta_{1}(t), \cdots, \eta_{m}(t)$ of the type described above for which the jacobian

$$
\left|\frac{\partial \Gamma^{j}}{\partial b_{k}}\right|_{\tau=b=0} \neq 0 \text {. }
$$

The equations

$$
\Gamma^{j}(\tau, b)=\gamma_{i},
$$$$
j=1, \cdots, m,
$$

have the initial solutions $\tau=b=0$, and at this solution the jacobian with re- 
spect to the $b_{j}$ is not zero by (4.12). So by the implicit functions theorem* there are functions $b=b_{\kappa}(\tau),(\kappa=1, \cdots, m)$, defined and of class $C^{\prime}$ for all small non-negative $\tau$, such that

$$
\Gamma^{j}(\tau, b(\tau)) \equiv \gamma_{j}, \quad j=1, \cdots, m .
$$

Let us define

$$
\mathrm{H}(\tau, b)=\Phi(\tau, b)+l_{\alpha} \Gamma^{\alpha}(\tau, b) \equiv \int_{C} H(z, \dot{z}) d t .
$$

Since the first variation of $\int H d t$ vanishes along $C$, we have

$$
\frac{\partial \mathrm{H}}{\partial b_{k}}=0 \quad \text { for } \quad \tau=b=0, \quad k=1, \cdots, m .
$$

By (3.10) and the mean value theorem, for $b=0$ we have

$$
\frac{\partial \mathrm{H}}{\partial \tau}=-\int_{t_{0}-\delta}^{t_{0}} \Omega_{H}(z(t), \dot{z}(t), r) d t=-\delta \Omega_{H}(z(\bar{t}), \dot{z}(\bar{t}), r)
$$

for some $\bar{t}$ between $t_{0}-\delta$ and $t_{0}$. Combining (4.16) and (4.17) we have

$$
\frac{d}{d \tau} \mathrm{H}(\tau, b(\tau))=\frac{\partial \mathrm{H}}{\partial \tau}+b_{\alpha}^{\prime}(\tau) \frac{\partial \mathrm{H}}{\partial b_{\alpha}}=\frac{\partial \mathrm{H}}{\partial \tau}<0
$$

for $\tau=0$. But by (4.14) and (4.15),

$$
\frac{d}{d \tau} \mathrm{H}(\tau, b(\tau))=\frac{d}{d \tau} \Phi(\tau, b(\tau)) .
$$

Now by (4.18) and (4.19) we see that

$$
\mathcal{F}\left(C_{\tau, b(\tau)}\right)=\Phi(\tau, b(\tau))<\Phi(0, b(0))=\Phi(0,0)=\mathcal{F}(C)
$$

for all small positive $\tau$, while by (4.14)

$$
G^{j}\left(C_{\tau, b(r)}\right)=\Gamma^{j}(\tau, b(\tau))=\gamma_{j} .
$$

This contradicts the minimizing property of $C$, and establishes the theorem.

5. Dresden's corner condition: the non-parametric form. In order that the proof of Theorem 1 shall apply also to integrands $F\left(z, z^{\prime}\right), G^{j}\left(z, z^{\prime}\right)$ arising by (1.1), it is only necessary to verify that $z^{0^{\prime}}(t)>0$ along each of the comparison curves $C_{\tau, b}$ used. If $C$ has a class $D^{\prime}$ representation $z^{i}=z^{i}\left(z^{0}\right)$,

* The fact that $\Gamma^{i}$ is defined only for $\tau \geqq 0$ does not prevent our use of the theorem; we could for example extend the range of definition of $\Gamma^{j}$ as a function of $\tau$ and then finally disregard the extension. 
$(i=1, \cdots, q)$, then on identifying the parameter $t$ with $z^{0}$ we have $\dot{z}^{0}(t) \equiv 1$ along $C$. But along $C_{\tau, b(\tau)}$ we have

$$
\frac{d}{d t} z^{0}(t, \tau, b)=\dot{\zeta}_{\alpha}{ }^{0}(t)+b_{\alpha} \dot{\eta}_{\alpha}{ }^{0}(t)=1+b_{\alpha} \dot{\eta}_{\alpha}{ }^{0}(t) \geqq 1-|b| \max \left|\dot{\eta}^{0}(t)\right|>\frac{1}{2}
$$

if $|b|$ be small enough. So the comparison curves used, for which $|b|$ is near 0 , are allowable, and the proof applies to the non-parametric case also. Recalling (3.15), we have the following result:

THEOREM 2. Assume the following conditions satisfied:

(5.1) The curve $y=y(x),\left(x_{1} \leqq x \leqq x_{2}\right)$, is interior to $S$ and gives a strong relative minimum to the integral

$$
\mathcal{F}[y]=\int_{x_{1}}^{x_{2}} f(x, y, \dot{y}) d x
$$

in the class of all $D^{\prime}$ curves which join $\left(x_{1}, y\left(x_{1}\right)\right)$ to $\left(x_{2}, y\left(x_{2}\right)\right)$ and give assigned values $\gamma_{j}$ to the integrals

$$
G^{j}[y] \equiv \int_{x_{1}}^{x_{2}} g^{j}(x, y, \dot{y}) d x, \quad j=1, \cdots, m .
$$

(5.2) $y=y(x)$ has a corner at $x_{0}$.

(5.3) The curve $y=y(x)$ is normal.

(5.4) $1, l_{1}, \cdots, l_{m}$ are the isoperimetric constants, and

Then

$$
h\left(x, y, y^{\prime}\right) \equiv f\left(x, y, y^{\prime}\right)+l_{\alpha} g^{\alpha}\left(x, y, y^{\prime}\right) .
$$

$$
\omega_{h}\left(x_{0}, y\left(x_{0}\right), y^{\prime}\left(x_{0}-0\right), y^{\prime}\left(x_{0}+0\right)\right) \leqq 0 .
$$

UNIVERSITY OF VIRGINIA,

Charlottesville, Va. 